\title{
EDITORIAL
}

\section{Tuberculosis and HIV co-infection: do we have a surveillance system in Europe?}

\author{
E. Pontali*, M.B. Pasticci ${ }^{\#}$, A. Matteelli ${ }^{\mp}$, F. Baldelli ${ }^{\#}$ and G.B. Migliori ${ }^{+}$
}

. $s$ there any reason why a clinician should be interested in knowing about the state of the art in tuberculosis (TB) and HIV co-infection surveillance in Europe?

A question like, "Is TB-HIV co-infection a relevant issue?" is probably easier to answer, at least at a global level: TB-HIV coinfection is responsible for almost 400,000 deaths every year and TB is by far the major killer of HIV-infected persons, being responsible for over a quarter of the global burden of HIVassociated deaths; people living with HIV / AIDS infected with Mycobacterium tuberculosis are at 20-30 times greater risk of developing TB compared with HIV-uninfected persons [1].

And in Europe? To control TB and HIV co-infection, which, together with multidrug-resistant TB, is today the hardest obstacle to overcome in curbing the TB epidemic, the World Health Organization (WHO) promotes a specific package, included in the second element of the Stop TB Strategy [2]. Developed in 2004 by WHO and UNAIDS (Joint United Nations programme on HIV/ AIDS), this package is based on the collaboration between TB and $\mathrm{HIV} /$ AIDS national programmes, and advocates 12 collaborative activities that are summarised in table 1 [3].

The strategy is comprehensive: in spite of its age, the document still maintains its validity, as demonstrated by the modest changes proposed for its revision, which is currently almost complete $[4,5]$.

The activities summarised in table 1 as A2 and A4 are those that allow us to understand the epidemiology of the TB-HIV coinfection (for instance, to evaluate trends) and to take adequate public health actions whenever necessary. It is worth mentioning that the activity A4 represents a much bigger "umbrella" than that of $\mathrm{A} 2$.

Then what is the best monitoring and evaluation approach to these activities? Looking at the problem through the eyes of a clinician, we see that there are usually two scenarios.

In TB clinics, TB cases are systematically tested for HIV infection. When co-infection is diagnosed, patients are referred to

*Dept of Infectious Diseases, Galliera Hospital, Genoa, "Infectious Disease Section, Dept of Experimental Medicine and Biochemical Sciences, University of Perugia, Perugia, "Dept of Infectious Diseases, Brescia University Hospital, Brescia, and ${ }^{+}$WHO Collaborating Centre for Tuberculosis and Lung Diseases, Fondazione S. Maugeri, Care and Research Institute, Tradate, Italy.

CORRESPONDENCE: G.B. Migliori, WHO Collaborating Centre for Tuberculosis and Lung Diseases, Fondazione S. Maugeri, Care and Research Institute, Tradate, Italy. E-mail: giovannibattista. migliori@fsm.it (or co-managed in collaboration with) the HIV/AIDS clinic to ensure that anti-retroviral (ARV) drugs and cotrimoxazole prophylaxis (CTX) are initiated as soon as possible. In addition, appropriate counselling and socio-psychological support are delivered, according to national standards. In the TB clinic the TB register is updated with the complete TB data and with information about HIV testing and, when appropriate, with information on ARV and CTX use.

In HIV clinics, people living with HIV / AIDS are protected from acquiring new $M$. tuberculosis infections through infection control measures, and progression to active disease is prevented by isoniazid preventive therapy (IPT), while active TB is diagnosed as early as possible through intensified case-finding (ICF) interventions. The HIV/AIDS register and reporting system should show if, and when, ICF and IPT practices are applied.

Unfortunately, many countries in the European region are still facing difficulties in taking care of this double burden, or even simply in having an idea of the current epidemiology of TBHIV co-infection [6].

In spite of the call made by WHO and UNAIDS for stronger integration between HIV and TB responses [7, 8], there has been very slow implementation of these recommendations in most countries, including those that are hardest hit by the dual epidemic [5].

The same recommendations from the global strategy for TBHIV control apply to Europe, too [9].

However, specific country needs in Europe vary considerably. In fact, there are countries in the European region that consider both diseases among top public health priorities (e.g. countries of the former Soviet Union like Belarus, Estonia, the Russian Federation and Ukraine, etc.) and others where neither of the two is a priority (e.g. Austria, Denmark, Germany and Slovakia) [9]. From a European Centre for Disease Prevention and Control (ECDC) perspective, countries in the European Union (EU) area usually fall into the lower burden group for TB-HIV co-infection [6]. Nevertheless, according to WHO recommendations [3], surveillance of HIV prevalence among newly diagnosed TB cases should be carried out in all countries, as it is the most pivotal indicator of the countries' need for more extensive activities to control the co-infection.

As regular reporting channels for data on the TB-HIV coinfection surveillance are weak, the ECDC commissioned two studies to collect and analyse all available information on 


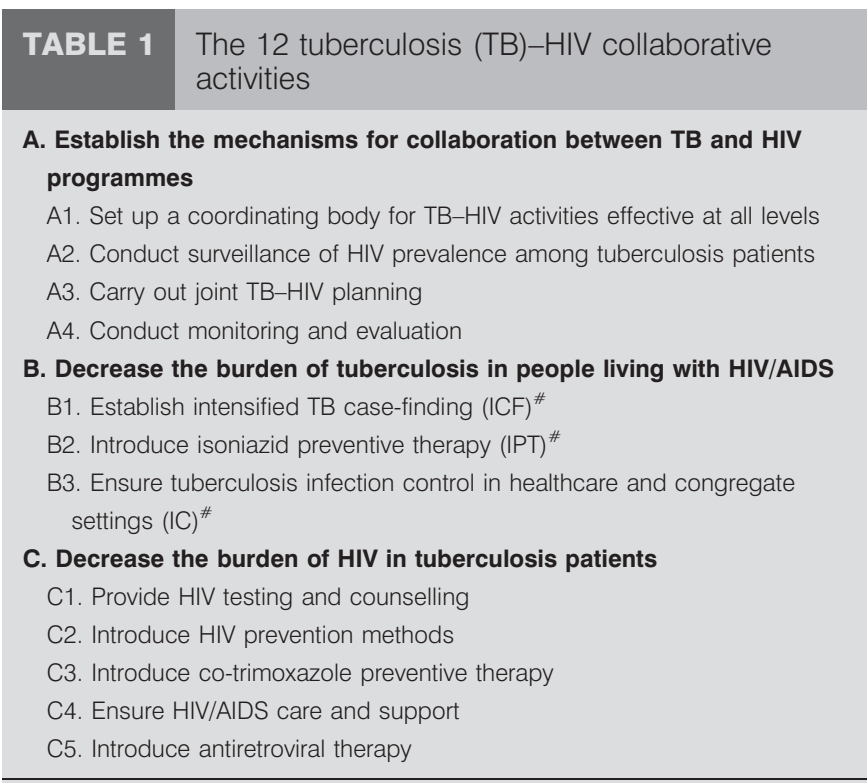

\#: ICF, IPT and IC are presently identified as "the three Is". Reproduced from [3] with permission from the publisher.

current TB-HIV surveillance practices and to assess the burden of the co-infection in Europe [6]. The study by KRUIJSHAAR et al. [10] collected information on TB-HIV surveillance practices in Europe.

The good news is that, in contrast to an earlier study that reported countries often did not have a national surveillance system to monitor HIV among TB patients [11], a large proportion of countries (72\%) have, as of today, such a system in place. However, seven of 25 responding EU/European Economic Area (EEA) countries still missed this target. Moreover, further efforts are needed to assess and improve the surveillance system where it is already in place and in use. The level of HIV testing in TB patients is low in some countries, despite national recommendations for routine testing: it should be further promoted and testing levels require regular monitoring in all countries. This is of crucial importance, particularly in EU/EEA countries where ARV treatment is (relatively) easily accessible.

The study by PIMPIN et al. [12] tries to fill a gap in the prevalence of HIV infection among newly diagnosed TB cases in EU/EEA countries. Despite the capacity to retrieve a large number of studies, the difficulty of the task is witnessed by the great heterogeneity among the studies as far as the geographical distribution, the period of observation and the adopted methodology are concerned, so that comparisons between countries and temporal trends need to be taken with some caution. Yet, increasing trends in HIV prevalence rates were observed in the Baltic States as well as Western EU countries, the Czech Republic and Romania, confirming that, even in countries where highly active ARV therapy is available and accessible, TB represents a persistent and increasing threat. However, the speculation that the increasing or decreasing trends of HIV infection among $\mathrm{TB}$ cases may be due to variations in the proportion of foreign-born people and the effectiveness of control measures among intravenous drug users should be taken with caution, as these data derive from a very limited number of studies. One interesting suggestion from this systematic review is that surveillance systems may under-represent marginalised populations and that efforts for identifying, treating and raising awareness for both diseases should be considered by countries with high levels of TB-HIV co-infection in these populations [12]. This need is reinforced by the evidence from the paper by KRUIJSHAAR et al. [10] that many TB patients are still not tested for HIV.

In Western European countries, TB-HIV activities are managed by clinical departments, as they have sufficient resources and capacity to follow-up these cases individually.

What is the capacity of TB and infectious diseases departments to ensure that adequate monitoring and evaluation of TB-HIV collaborative activities are carried out? In Italy, infectious diseases departments are taking care of co-infected cases. The experience of major departments in Brescia, Genova and Perugia is that the information on TB-HIV co-infected cases is inconsistently available from local registers, but in the absence of an organised information flow, data are not used to inform on the epidemiological situation and to plan further strengthening of the activities. For example, at the infectious diseases department of Perugia, a teaching hospital in central Italy, where 50 TB-HIV co-infected patients have been diagnosed and treated (46\% of them being foreign-born) among the cohort of 354 TB patients followed up since the introduction of ARV (in 1987), the group A activities (table 1) are not in the responsibility/capacity of the clinical centre, while the others are carried out but they are not used to generate reports.

These latter examples from Italy represent an approach that seems suitable for low burden countries. Nevertheless, in Italy and, probably, in other Western European Countries, surveillance is still behind schedule. The finding that seven EU countries (Italy included) are not implementing it calls for further advocacy on this important issue. Effective surveillance allows for early detection of any warning in the TB-HIV epidemiology and calls for adequate action, in this case represented by implementation of the whole 12-point package (of which monitoring and evaluation is part).

Better information on how TB and HIV activities are performed to ensure that the single patient affected by two diseases is cared for in a quality manner is useful for the European clinician, as this evidence will allow him/her to request for further human and financial resources, to better equip clinical departments, to improve advocacy, and to plan and implement the necessary operational research activities to respond to the (many) still unanswered questions.

This is a win-win opportunity that clinicians and public health officers should tackle together.

The European Respiratory Journal's leadership in the field of TB is confirmed once more by the two studies published in this issue by KRUIJSHAAR et al. [10] and PIMPIN et al. [12] on behalf of the ECDC.

Further evidence on how surveillance and the other TB-HIV collaborative activities are performed in Europe is needed. 


\section{STATEMENT OF INTEREST}

None declared.

\section{REFERENCES}

1 World Health Organization. Multidrug and Extensively Drugresistant TB (M/XDR-TB): 2010 Global Report on Surveillance and Response (WHO/HTM/TB/2010.3). Geneva, World Health Organization, 2010.

2 Raviglione MC, Uplekar MW. WHO's new Stop TB Strategy. Lancet 2006; 367: 952-955.

3 World Health Organization. Interim Policy on Collaborative TB/ HIV Activities. Geneva, World Health Organization, 2004.

4 World Health Organization. Strategic and Technical Advisory Group For Tuberculosis (STAG-TB), Report of the Tenth Meeting, 27-29 Sept 2010 (WHO/HTM/TB/2010.18). Geneva, World Health Organization, 2010. Available from: www.who.int/entity/tb/ advisory_bodies/stag_tb_report_2010.pdf

5 Sester M, Giehl C, McNerney R, et al. Challenges and perspectives for improved management of HIV/Mycobacterium tuberculosis coinfection. Eur Respir J 2010; 36: 1242-1247.
6 European Centre for Disease Prevention and Control/WHO Regional Office for Europe. Tuberculosis surveillance in Europe 2009. Stockholm, European Centre for Disease Prevention and Control, 2011. Available from: http://ecdc.europa.eu/en/publica tions/Publications/Forms/ECDC_DispForm.aspx?ID=660.

7 World Health Organization. The Global Plan to Stop TB, 20062015. Geneva, World Health Organization, 2006.

8 WHO, UNAIDS, PEPFAR. A Guide to Monitoring and Evaluation for Collaborative TB/HIV Activities - 2009 Revision. Geneva, World Health Organization, 2009.

9 De Colombani P, Banatvala N, Zaleskis R, et al. European framework to decrease the burden of TB/HIV. Eur Respir J 2004; 24: 493-501.

10 Kruijshaar ME, Pimpin L, Abubakar I, et al. The burden of TB-HIV in the EU: how much do we know? A survey of surveillance practices and results. Eur Respir J 2011; 38: 1374-1381.

11 Lazarus JV, Olsen M, Ditiu L, et al. Tuberculosis-HIV co-infection: policy and epidemiology in 25 countries in the WHO European region. HIV Med 2008; 9: 406-414.

12 Pimpin L, Drumright LN, Kruijshaar ME, et al. Tuberculosis and HIV co-infection in European Union and European Economic Area countries. Eur Respir J 2011; 38: 1382-1392. 